\title{
Highlights of the 2009 Society of Vascular Surgery annual meeting
}

\author{
Michael Belkin, MD
}

The annual meeting for the Society of Vascular Surgery (SVS) took place June 11 to 14, 2009, in Denver, Colorado. A highlight of the meeting was the presidential address of $\mathrm{G}$. Patrick Clagett, who raised the question: "Does Vascular Surgery Cost Too Much?" He reviewed the staggering cost of healthcare in our country and stressed that the rate of growth in healthcare expenditures is not sustainable and that the huge disparity in healthcare spending among a variety of industrialized nations has not translated into better healthcare outcomes. Dr Clagett described the unusual paradox that higher healthcare spending has often been associated with lower quality of care and slightly worse outcomes. He went on to say that a significant proportion of President Obama's \$1.1 billion economic stimulus package has gone to the National Institutes of Health, Agency for Healthcare Research and Quality, and Department of Health and Human Services, specifically for comparative effectiveness research (CER). Although not specifically stated, CER will have clear economic implications, and it is hoped that these programs will reduce variation in practice and save money. Dr Clagett stressed that the SVS has been active in CER through its Practice Guidelines and Reporting Standards Committees. The SVS Vascular Registry has also been an important contribution. Finally, Dr Clagett charged the members of the SVS to be more actively involved in CER. He cited that of all specialty groups managing peripheral vascular disease, we are the most unbiased because we are able to offer all treatment modalities, and are thus best positioned to offer high-quality CER. He concluded by stating, "Finally, these mandates are clearly spelled out in our mission statement. Integrity is our middle name. This is the right thing to do for our membership, for our patients, and for the public.",

K. Wayne Johnston, MD, from Toronto, was presented the SVS Lifetime Achievement award.

Among his many outstanding contributions were his term as president of the SVS from 2007 to 2008 and his role as Editor-in-Chief of the Journal of Vascular Surgery.

\section{CAROTID ARTERY OCCLUSIVE DISEASE}

White and colleagues, ${ }^{1}$ from the SVS Outcomes Committee, reported the results from the SVS Vascular Registry,

\footnotetext{
From the Division of Vascular and Endovascular Surgery, Brigham and Women's Hospital, Harvard Medical School, Boston, Mass.

Address for reprints: Michael Belkin, MD, Brigham and Women's Hospital, Harvard Medical School, Boston, MA. (E-mail: mbelkin@partners.org).

J Thorac Cardiovasc Surg 2009;138:1257-61

0022-5223/\$36.00

Copyright $₫ 2009$ by The American Association for Thoracic Surgery doi:10.1016/j.jtcvs.2009.07.057
}

focusing on the comparison of carotid artery stenting for atherosclerotic carotid artery disease versus nonatherosclerotic carotid artery disease. This is a large, voluntary, self-reported registry. Of 4017 patients with carotid artery stents who are listed in the registry, $72 \%$ were treated for atherosclerosis and $28 \%$ were treated for nonatherosclerosis, including restenosis $(76 \%)$, radiation-induced disease $(16 \%)$, and others $(8 \%)$. Patients with atherosclerosis were older and more often male, and had a higher incidence of coronary disease, previous myocardial infarction, and other comorbidities. Patients without atherosclerosis had a higher incidence of amaurosis fugax, smoking, and cancer history. Between the 2 groups, there were no statistically significant differences in the rate of death, stroke, or myocardial infarction at 30 days $(3.03 \%$ for the atherosclerosis group vs $2.72 \%$ for the nonatherosclerosis group). There were also no differences in outcomes based on gender or symptomatic status. The authors concluded that although patients with atherosclerosis appeared to be sicker because of additional comorbidities, they did not have poor outcomes compared with patients without atherosclerosis when treated with carotid artery stenting.

Schor and coworkers, ${ }^{2}$ from the University of Medicine and Dentistry of New Jersey in Newark, studied the impact of prior cervical irradiation on carotid artery stenting. They reviewed the results of 37 patients who underwent carotid artery stenting after a history of cervical irradiation. They compared these patients with a larger cohort without previous radiation. The patients did not differ with respect to age, gender, smoking status, or symptoms. Radiated patients had a lower incidence of hypertension, diabetes, and coronary disease. The 30-day stroke, death, or myocardial infarction rate was similar in both groups $(5.4 \%$ of irradiated patients vs $5.9 \%$ of nonirradiated patients). In-stent restenosis in follow-up, however, was higher in patients after radiation therapy at $76 \%$ versus $21.6 \%(P=.01)$. Some $18.9 \%$ of radiated patients required an additional intervention versus $4.7 \%$ of nonirradiated patients $(P=.002)$. The authors concluded that although periprocedural complications were not increased for carotid artery stenting in patients with prior cervical radiation, the durability of carotid artery stenting was impaired and the need for additional revascularization procedure was increased in these patients.

AbuRahma and associates, ${ }^{3}$ from Charleston, West Virginia, studied the outcomes of primary carotid stenting versus stenting for recurrent carotid stenosis after previous endarterectomy. Of 180 patients in the study, 68 had primary carotid artery stents versus 112 after a previous carotid endarterectomy. Clinical characteristics were comparable between the 2 groups, and follow-up averaged 25 months. 
The technical success rate was $100 \%$. Combined perioperative stroke, death, and myocardial infarction rates were $7.4 \%$ for the primary group versus $0.9 \%$ for the recurrent carotid stenosis group $(P=.03)$. Stroke-free rates remained significantly different up to 4 years. Freedom from in-stent restenosis was high in both groups. The authors concluded that carotid artery stenting was safer for restenotic lesions than primary carotid artery plaques.

\section{ABDOMINAL AORTIC ANEURYSMS}

Lederle and colleagues ${ }^{4}$ reported the 2-year results of the highly anticipated OVER trial. This Veterans Administration Hospital-based trial randomized 881 patients $(99.3 \%$ were male) to open versus endovascular aneurysm repair (EVAR) (with any Food and Drug Administration-approved device) of infrarenal aortic aneurysms over a 9-year period. Mortality was the primary end point. Thirty-day mortality was low in both groups but significantly less in the EVAR group $(0.2 \%$ vs $2.3 \% ; P=.006)$. Mortality at 2 years follow-up did not differ (EVAR 7\% vs open $9.4 \% ; P=.19$ ). Major morbidity at 1 year did not differ (EVAR $4.4 \%$ vs open $4.3 \%$ ). Quality of life and erectile dysfunction did not differ between the groups. The authors pointed out that the mortality reported in this trial is lower than in previous randomized trials. Although there was a perioperative survival advantage with EVAR, it was not sustained in follow-up.

Greenberg and colleagues ${ }^{5}$ reported the intermediate results of a US multicenter trial of fenestrated endograft repair for juxtarenal abdominal aortic aneurysms. These fenestrated grafts and applied technologies are of obvious interest and import for thoracic endovascular grafting involving the aortic arch. Six centers enrolled 30 patients with juxtarenal aneurysms with short proximal necks, which would preclude conventional endovascular repair. A total of 77 visceral vessels were accommodated by fenestrations in these 30 patients. The most common designs accommodated 2 renal arteries and a superior mesenteric artery $(66.7 \%)$. All endografts were successfully implanted without the acute loss of any visceral arteries. Twenty-three of the patients were available for a 24-month follow-up. There were no aneurysm-related deaths, ruptures, or conversions to open procedures throughout 24 months. There were no type 1 or 3 endoleaks observed at any time point. There were no cases of aneurysm growth greater than $5 \mathrm{~mm}$ in diameter. Eight patients experienced some form of renal event, including 4 renal artery stenoses, 2 renal artery occlusions, and 2 renal infarcts. Among these patients, 5 underwent secondary interventions. No patient required dialysis for renal failure. The authors concluded that fenestrated endovascular grafts are feasible in centers with extensive experience in EVAR. They were encouraged that the results achieved were concordant with other studies reported from single centers of excellence.

Beck and associates ${ }^{6}$ presented a series of 18 patients from Athens, Greece, who were treated with fenestrated and branched endografts for repair of juxtarenal and pararenal abdominal aortic aneurysms after previous open infrarenal aortic aneurysm repair. The mean time from the previous operation was 8.5 years. The mean number of fenestrations per patient was 3 . All patients but 1 were treated completely by endovascular surgery. The mean operative time was 215 minutes, with a blood loss of $560 \mathrm{~mL}$. Perioperative complications occurred in 2 patients. After a follow-up of 23 months, the cumulative primary patency was $95 \%$ (53/56 vessels). The authors concluded that endovascular treatment of juxtarenal and pararenal aneurysms after previous aortic reconstruction is a viable alternative to open repair with a high success rate and low reintervention rate.

Mehta and associates, ${ }^{7}$ from Albany Medical College, studied the implications of endoleaks on aneurysm sac pressure after endovascular repair of elective and ruptured aortic aneurysms. They reported a series of 480 patients who underwent infrarenal EVAR with placement of the CardioMEMS Endosure wireless pressure sensor within the excluded aneurysm sac. The authors documented (CardioMEMS, Atlanta, $\mathrm{Ga})$ that in patients with type 2 endoleaks $(102,21 \%)$, there were marked elevations of all pressures compared with patients with no endoleaks. Treatment of type 2 endoleaks led to a reduction in these pressure indices to those of patients without endoleaks. Patients with type 1 endoleaks had significant elevations of pressures that approximated pre-exclusion aneurysm sac pressures. Similarly, these pressures decreased significantly after the treatment of type 1 endoleaks. The authors concluded the presence of type 1 and 2 endoleaks can be predicted by the evaluation and follow-up of aortic aneurysm sac pressures. Successful treatment of endoleaks can be predicted by normalization of these sac pressures.

\section{THORACIC ENDOVASCULAR STENT GRAFTING}

Rakhlin and colleagues, ${ }^{8}$ from the University of Pennsylvania, reported on the use of thoracic endovascular stents in the management of acute complicated type B dissections and compared the results in patients with malperfusion syndrome versus rupture. From 2004 to 2008, 43 patients with acute complicated type B dissections underwent thoracic endovascular stent grafting and were included in this evaluation. Twenty-six patients $(60 \%)$ presented with malperfusion, 22 patients $(51 \%)$ presented with rupture, and 5 patients (11\%) presented with both. In those with malperfusion, renal malperfusion occurred in 17 patients $(65 \%)$, visceral malperfusion occurred in 17 patients $(65 \%)$, and lower extremity malperfusion occurred in 14 patients $(54 \%)$. The average patient follow-up was 16 months. Excellent technical and clinical results were achieved in both groups. Patients who were treated for rupture presented and were treated earlier. Endograft use and deployment were comparable between the 2 groups. One-year survival was $94 \%$ in both groups. Thoracic endovascular grafting alone was effective in treating aortic rupture in 21 patients $(95 \%)$, whereas only 15 patients 
$(58 \%)$ in the malperfusion group could be completely treated with stent grafting. Eleven patients $(42 \%)$ in the malperfusion group required an adjunctive procedure (almost all endovascular) to restore end-organ perfusion. The authors stressed that although both malperfusion and rupture complicating type B aortic dissections can be successfully treated with thoracic endografting, the treatment must be customized to each patient's presentation to achieve these results.

Mousa and colleagues, ${ }^{9}$ of the University of Medicine and Dentistry of New Jersey, New Brunswick, used the National Inpatient Sample database to evaluate the outcomes and hospital use of thoracic endovascular aneurysm repair (TEVAR) versus open thoracic repair in the management of acute aortic trauma. By using the National Inpatient Sample from 2005 to 2006, they identified 1561 patients with thoracic aortic injury; 480 patients underwent emergency surgical intervention, with 245 patients being treated with open thoracic repair and 235 patients being treated with TEVAR. Mortality after open repair was significantly greater $(14.32 \%)$ than after TEVAR $(8.52 \% ; P=.046)$. Patients treated with open repair were 2.2 times likely to have pulmonary complications. After adjustment for age, gender, and comorbidities, mortality after open repair was greater (odd ratio $=2.9 ; 95 \%$ confidence interval, 1.42-5.75) than after TEVAR. Length of stay after open repair was significantly longer at 23.8 days versus 13.5 days $(P=.0029)$ for TEVAR. Open repair was significantly more expensive than TEVAR $(\$ 83,027$ vs $\$ 57,092 ; P=.0214)$. The authors concluded that the implementation of TEVAR has been associated with an increasing number of patients receiving intervention for traumatic aortic injury along with decreased mortality and resource use. Further implementation of TEVAR for aortic trauma may improve future outcomes and reduce hospitalization use.

Tefera and colleagues, ${ }^{10}$ from the University of Wisconsin, presented a further comparison on hospital cost and clinical outcomes for open versus endovascular thoracic aortic aneurysm repair in a single-center study. From November 2006 to May 2008, 60 patients underwent repair of thoracic aortic aneurysms with either open surgery (35) or TEVAR (25). An average of 1.8 stent grafts were used per patient. Morbidity and mortality were similar in both groups. There was 1 case of paraparesis in the TEVAR group and 1 death in the open surgical group. Five secondary interventions were performed for type 1 endoleaks after TEVAR. Hospital stay was significantly shorter and the nursing cost was lower for the TEVAR group. Total hospital cost and operating cost were significantly higher for the TEVAR group. The net margin was significantly negative for the TEVAR group at a loss of $\$ 20,222$ per case. In their discussion, the authors raised the question as to whether the increased cost associated with TEVAR could be justified if major advantages in terms of morbidity and mortality cannot be documented.

Bismuth and coworkers ${ }^{11}$ from Methodist Debakey Heart and Vascular Center, Houston, reported the results of a series of 20 patients who were monitored with transcranial Doppler during endovascular repair of the thoracic aorta. ${ }^{11}$ The highest number of cerebral microembolic signals occurred with pigtail catheter placement during the diagnostic stage and by device placement during the deployment stage. During the diagnostic stage, an average of 8.6 microembolic signals occurred, whereas during the deployment stage, 45 right intracranial signals and 43 left intracranial signals occurred. There was no correlation between the number of microembolic signals and postoperative stroke, transient ischemic attack, arch type, landing zone, or coverage of the subclavian artery. The authors concluded that transcranial Doppler may prove to be an important adjunct in thoracic endografting to identify which steps and procedures are most likely to provoke emboli and to compare endografts and their capacity to generate microemboli.

Shah and associates, ${ }^{12}$ from New York University, studied the results of thoracic endovascular grafting in female patients. TEVAR was performed in 51 patients ( 29 female, $56 \%$ ) for a variety of aortic pathologies. The thoracic aortic diameter was larger in women than in men (59 vs $47 \mathrm{~mm}$ ). TEVAR was more likely to be performed on an urgent basis in women compared with men. Women were more likely to require a common iliac artery conduit compared with men $(48.3 \%$ vs $20 \%, P<.05)$. There was a trend toward an increased paraplegia rate in women $(10.3 \%$ vs $4.8 \%)$. The authors thought this might be related to an increase in total length of aortic coverage in women compared with men $(18.2$ vs $15.2 \mathrm{~cm}, P<.05)$. The authors concluded that prophylactic measures to prevent spinal ischemia should be considered and that the length of coverage should be minimized in female patients.

Pisimisis and colleagues ${ }^{13}$ presented a 2-institution evaluation on the incidence and risk factors of renal dysfunction after thoracic endovascular aortic repair. Between 1998 and 2008, 175 patients underwent 210 TEVAR procedures for various aortic pathologies at 2 tertiary institutions. Aortic pathologies included 103 aneurysms, 72 dissections, 21 transections, and 14 penetrating ulcers. Mean patient age was 70 years, with a median preoperative estimated glomerular filtration rate (eGFR) of $65 \mathrm{~mL} / \mathrm{min} / 1.73 \mathrm{~m}^{2}$. Average procedural contrast load was $108.7 \pm 70 \mathrm{~mL}$. Median eGFR at 24 hours and 30 days was $69 \mathrm{~mL} / \mathrm{min} / 1.73 \mathrm{~m}^{2}$ and $67 \mathrm{~mL} / \mathrm{min} / 1.73 \mathrm{~m}^{2}$, respectively. The rates of acute renal injury, acute renal failure, and hemodialysis were $15.7 \%, 3.1 \%$, and $0.5 \%$, respectively. Risk factors associated with a decrease in eGFR at 24 hours were procedural blood loss, packed red blood cell transfusion, lengthy procedures, open surgical conversion, and several stents deployed. At 1 month, prognostic factors included increasing age, preoperative rupture, infrarenal abdominal aortic aneurysm, zone 0-2 deployment, larger proximal neck diameter, and shorter intensive care unit and hospital stays. Factors that proved to be protective of renal function were obesity, 
trauma as an underlying pathology, and a stable hemodynamic course. The authors noted in their discussion that the incidence of renal failure in this cohort was lower than that compared with previous smaller series. They attributed this in part to the routine use of intravascular ultrasound during the procedure and reduced contrast load.

\section{OPEN REPAIR OF THORACIC AND THORACOABDOMINAL ANEURYSMS}

To provide a contemporary view of the results of open repair of thoracoabdominal aortic aneurysms in the United States, Oderich and colleagues, ${ }^{14}$ from The Mayo Clinic, queried the results from the National Inpatient Sample for open non-urgent repair of thoracoabdominal aortic aneurysms from 2000 to 2006. A total of 5776 open repairs were identified during the 7-year period. The median age was 70 years, and $43 \%$ of the patients were women. Major comorbidities included hypertension (56\%), chronic pulmonary disease $(35 \%)$, chronic renal insufficiency $(11 \%)$, and prior myocardial infarction $(8 \%)$. Postoperative in-house mortality was significant at $16 \%$. Postoperative complications included acute renal failure ( $25 \%$ ), pulmonary complications $(10 \%)$, mesenteric ischemia $(3 \%)$, and myocardial infarction $(3 \%)$. Acute renal failure was the main predictor of in-hospital mortality, with an odds ratio of 3.5. Independent factors associated with postoperative acute renal failure were increased age, patient comorbidity index, chronic renal insufficiency, and surgery in a rural or nonteaching hospital. The authors concluded that open surgical repair of thoracic aortic aneurysms continues to carry a high postoperative mortality. They believe the data presented serves as an important benchmark against which new endovascular and hybrid approaches can be compared for the treatment of thoracic aortic aneurysms.

The group from the University of Texas Health Science Center gave a number of presentations and posters on various aspects of open thoracic and thoracic aortic aneurysm repair. One such presentation was by Miller and colleagues, ${ }^{15}$ who focused on respiratory morbidity after thoracic aortic aneurysm repair. Between 1991 and 2008, 1443 descending thoracic or thoracic aortic aneurysms were repaired with open surgery. The mean age of patients was 68 years, and $37 \%$ were female. A total of 432 patients $(30 \%)$ required mechanical ventilation more than 72 hours after surgery. Significant risk factors for prolonged mechanical ventilation included a preoperative forced expiratory volume at 1 second less than $80 \%$ of predicted, a decreased GFR, emergency presentation, rupture, and chronic obstructive pulmonary disease. The only intraoperative variable associated with prolonged mechanical ventilation was prolonged aortic crossclamp time. Mortality among patients with prolonged ventilation was $31 \%$ versus $10 \%$ of those patients without prolonged ventilation. The authors concluded that ventilator failure after open thoracic surgery occurs predominantly in high-risk patients with poor preoperative pulmonary and renal function, as well as those presenting for urgent procedures. Mortality is significantly increased in these patients. Estrera and colleagues ${ }^{16}$ presented an additional poster from this group examining the factors predictive of neurologic deficit and mortality in contemporary practice. Immediate neurologic deficits occurred postoperatively in 37 of 1443 patients $(2.5 \%)$. Significant predictors were type 2 thoracoabdominal aortic aneurysms and decreased preoperative eGFR. The use of cerebrospinal fluid drainage and distal aortic perfusion as an adjunct reduced neurologic morbidity by two thirds (odds ratio $0.33, P<.002$ ). Significant predictors of mortality were decreased eGFR, preoperative rupture, peripheral vascular disease, coronary artery disease, and type 2 or 3 aortic aneurysm repairs. Miller and colleagues ${ }^{17}$ examined 1067 of 1443 patients who had distal aortic perfusion during thoracoabdominal aortic aneurysm repair. The authors noted that in September of 2006, they adopted a side-arm femoral cannulation technique as opposed to a direct femoral cannulation in an effort to eliminate leg ischemia during cannulation time. Their hypothesis is that leg ischemia contributes to decreased renal function postoperatively. The authors demonstrated that side-arm cannulation was associated with an approximate $50 \%$ reduction in peak postoperative creatinine in patients with a preoperative GFR less than $70 \mathrm{~mL} / \mathrm{min} / 1.73 \mathrm{~m}^{2}$. The authors concluded that side-arm cannulation was associated with a clinically important and statistically significant reduction in postoperative creatinine levels in patients with low preoperative GFRs. They hypothesized that reduced skeletal muscle ischemia is the likely mechanism.

\section{References}

1. White RA, Sicard GA, Zwolak RM, et al. SVS Vascular Registry for carotid procedures: comparison of CAS outcomes for atherosclerotic carotid artery disease with non-atherosclerotic carotid artery disease. J Vasc Surg. 2009;49(Suppl May):S12.

2. Schor JA, Kapadia I, Jamil Z, et al. Prior cervical radiation predicts reduced durability of CAS. J Vasc Surg. 2009;49(Suppl May):S17.

3. AbuRahma AF, Abu-Halimah S, Bensenhaver J, et al. Primary carotid artery stenting versus carotid artery stenting for post- restenosis: early and late outcomes. J Vasc Surg. 2009;49(Suppl May):S14.

4. Lederle FA, Freischlag JA, Kyriakides TC, et al. Two-year comparison of endovascular and open repair of abdominal aortic aneurysm. Abstract.

5. Greenberg RK, Sternbergh WC, Makaroun M, et al. Intermediate results of a US multicenter trial of fenestrated endograft repair for juxtarenal abdominal aortic aneurysms. Abstract.

6. Beck AW, Bos WTG, Vourliotakis G, et al. Fenestrated and branched endograft repair of juxta- and para-renal aneurysms after previous open aortic reconstruction. J Vasc Surg. 2009;49(Suppl May):S6.

7. Mehta M, Taggert JB, Roddy SP, et al. Implications of endoleaks on aneurysm sac pressures following endovascular repair of elective and ruptured aortic aneurysms. J Vasc Surg. 2009;49(Suppl May):S7.

8. Rakhlin EY, Szeto WY, Fairman RM, et al. Successful management of acute complicated type B dissections with TEVAR and adjunctive endovascular techniques: malperfusion versus rupture. J Vasc Surg. 2009;49(Suppl May):S4.

9. Mousa AY, Dombrovskiy VY, Coyle BW, et al. Thoracic aortic trauma: outcomes and resource utilization for endovascular vs. open repair. Abstract. New Brunswick, NJ

10. Tefera G, Hoch J, Mell M, et al. Hospital cost and clinical outcomes following open and endovascular thoracic aortic repair. Abstract. Madison, WI: University of Wisconsin Medical School. 
11. Bismuth J, Garami Z, Younes HK, et al. Transcranial Doppler monitoring is vital in thoracic endograft placement. J Vasc Surg. 2009;49(Suppl May):S18-9.

12. Shah TR, Bauer SM, Cayne NS, et al. TEVAR in female patients: increased aortic coverage is associated with a higher risk of spinal cord ischemia. J Vasc Surg. 2009;49(Suppl May):S19.

13. Pisimisis G, Kantor B, Khoynezhad A, et al. Incidence and risk factors of renal dysfunction after thoracic endovascular aortic repair. J Vasc Surg. 2009;49(Suppl May):S36.

14. Oderich GS, Rosero EB, Ricotta JJ, et al. In-hospital mortality of open thoracoabdominal aortic aneurysm repair in the united states prior to widespread use of hy- brid and endovascular branched techniques. J Vasc Surg. 2009;49(Suppl May) S55.

15. Miller CC, Estrera AL, Azizzadeh A, et al. Respiratory morbidity following thoracoabdominal aortic repair. J Vasc Surg. 2009;49(Suppl May):S35.

16. Estrera AL, Miller CC, Azizzadeh A, et al. Open repair of descending thoracic and thoracoabdominal aortic aneurysms: contemporary results. J Vasc Surg. 2009; 49(Suppl May):S35-6.

17. Miller CC, Estrera AL, Azizzadeh A, et al. Postoperative creatinine reduction with non-ischemic femoral arterial cannulation for thoracoabdominal aortic repair. $J$ Vasc Surg. 2009;49(Suppl May):S56. 